\title{
Double-layer polarization induced transitions in suspensions of colloidal rods
}

\author{
Short title : Double-layer polarization \\ Kyongok Kang and Jan K.G. Dhont \\ Forschungszentrum Jülich \\ Institute für Festkörper Forschung (IFF), Weiche Materie \\ D-52425 Jülich, Germany
}

\begin{abstract}
Polarization of electric double layers of colloidal rods due to an external alternating electric field is found to give rise to several phase/state transitions. Various phases and states are observed depending on the frequency and amplitude of the external electric field: (i) non-chiral nematic domains ( $N$-domains) in coexistence with an isotropic phase, (ii) a chiral-nematic phase in the presence of the $N$-domains, (iii) a chiral nematic phase, where now the $N$-domains are smaller and disconnected, and (iv) dynamic states where the chiral nematic is melted, in the presence of disappearing and forming $N$-domains. Beyond a critical frequency (in the $k H z$ range) the only stable state is the isotropic state. The phase/state diagram in the field-amplitude versus frequency plane is determined by means of polarization microscopy. In selected parts of the phase diagram, dynamic light scattering, electric birefringence and chiral-pitch measurements are performed to elucidate the nature of transition lines.
\end{abstract}

PACS numbers 47.20.Hw,64.60.-i,82.70.Dd 
Introduction The response of materials containing rod-like molecules to external electric fields has been studied extensively. These studies are almost entirely concerned with molecules that are either dielectrically polarizable or have a permanent dipole moment, such as thermotropic liquid crystals and electro rheological fluids. The response of thermotropic liquid crystalline materials to electric fields is due to dielectric polarization or the coupling of a permanent dipole of the molecules to the external field. An overview of the large body of literature on thermotropics in electric fields and a discussion of the mechanisms for these materials can be found in the book of de Gennes and Prost [1]. Double-layer polarization plays no role in thermotropics but can be important for lyotropic colloidal materials. Experiments on lyotropic colloidal materials are usually done at very high frequencies (in the $k H z-M H z$ range) [2], [3], [4]. For such high frequencies, the double layer can not respond and is therefore unaffected by the external field. In this case the core of the colloidal particles is dielectrically polarized. Polarization of electric double layers in lyotropic materials has rarely been addressed. There are no studies so far on phase/state transitions of colloidal suspensions in external alternating electric fields due to double-layer polarization. The present Letter describes phase/state transitions of rod-like colloids with extended double layers in oscillating electric fields with low frequencies (up to $5 \mathrm{kHz}$ ), which are due to interactions of polarized double layers and possibly the accompanied electro-osmotic flows.

A few experimental studies are reported on colloidal dispersions of rods at lower frequencies where interactions between polarized double-layers are important [5],[6]. In Ref.[5], relatively dilute dispersions (up to a few times the overlap concentration) of rod-like colloids (fd-virus suspensions) have been investigated by means of birefringence for various, very low ionic strengths. Mixtures of charged colloidal spheres and rods are studied in Ref.[6]. In both studies an "anomalous" alignment of the rods perpendicular to the electric field is found as soon as colloid-colloid interactions become relevant. The mechanism leading to this anomalous orientation is still 
unclear. Both direct interactions between polarized double layers and hydrodynamic interactions through electro-osmotic flows [7] can be important. A possible mechanism for anomalous orientation of single flexible rods is suggested in Ref.[8] : hydrodynamic friction of flexible rods is minimized when they orient perpendicular to the electric field. For the above mentioned experiments, however, interactions between colloids seem to be essential for the anomalous behaviour. These interactions are also responsible for the zigzag-patterns observed in suspensions of colloids and polyelectrolytes in very thin cells at high field strengths and low frequencies [9]. These patterns are due to confinement effects that are absent in the bulk experiments in Refs.[5],[6]. The experiments described in this Letter are also concerned with bulk properties.

Experimental Details The colloidal system we use consists of solutions of fd-virus particles at a low ionic strength (a TRIS/HCl buffer of $1.6 \times 10^{-4} M$, corresponding to a Debye length of $25 \mathrm{~nm}$ ). The ionic strength is set by the concentration of the buffer solution against which the suspensions are dialyzed for at least one day. Fd-viruses are stiff, rod-like colloids with a length of $880 \mathrm{~nm}$ and a core-thickness of $6.7 \mathrm{~nm}$. Two concentrations are systematically studied, $2.0 \mathrm{mg} / \mathrm{ml}$ and $2.8 \mathrm{mg} / \mathrm{ml}$. These concentrations are $\sim 30$ times larger than the overlap concentration. The phase behaviour of fd-virus suspensions at higher ionic strengths, larger than $5 \mathrm{mM}$, in the absence of an external field has been thoroughly investigated in Refs.[10],[11],[13]. At these higher ionic strengths a phase transition from isotropic to chiral nematic is observed at fd concentrations that monotonically increase with increasing ionic strength. A non-chiral nematic state is not observed. Extrapolation of the lower binodal concentrations for isotropic/chiral-nematic coexistence as a function of ionic strength as reported in Refs.[10],[11],[13] down to $1.6 \mathrm{mM}$ gives $3.2 \mathrm{mg} / \mathrm{ml}$. Surprisingly, we found a non-chiral nematic state above a fd concentration of $1.5 \mathrm{mg} / \mathrm{ml}$ (up to $4.0 \mathrm{mg} / \mathrm{ml}$, which is the largest concentration that we probed). No chiral-nematic state without an electric field could be found in the absence of an electric field at this low ionic strength. It thus seems that the large extent 
of the double layer screens the chiral structure of the core of the fd strand, and thus prevents the formation of a chiral nematic state.

In our experiments we use commercially available, custom-designed high resistance indiumtin-oxide (ITO) coated float glass of dimensions $40 \times 70 \mathrm{~mm}^{2}$. An amount of $400 \mu l$ of fdsuspension is loaded on a plate within a rectangular area $\left(40 \times 30 \mathrm{~mm}^{2}\right)$ surrounded with electric insulating film-spacer. The spacer thickness is typically $1.2-1.4 \mathrm{~mm}$. The precise sample thickness is determined from the radius of the circular area occupied by the suspension after the top plate is positioned. Radii of the sample volume are about $10 \mathrm{~mm}$, and measurements are done at the center of the sample to ensure a homogeneous electric field. A function generator produces an alternating sinusoidal waveform. A CCD camera is mounted on an inverted microscope to collect images of de-polarized light combined with Differential Interference Contrast (PlasDIC, Carl Zeiss). The use of differential interference enhances the contrast of images due to the presence of small-scale variations in refractive index within the sample, which result from the inhomogeneities that exist in coexistence regions. Since the dimensions of morphologies of interest are larger than about $10 \mu \mathrm{m}$, it is sufficient to collect images with $10 X$ objective lens. The field of view is large enough to image an appropriate portion of the sample $\left(900 \times 700 \mu m^{2}\right.$ with $1300 \times 1030$ pixels $)$. In addition, a Dynamic Light Scattering (DLS) and a birefringence set up are used with the above discussed home-made electric cell to probe dynamics and orientation in selected parts of the phase diagram.

Results and Discussion Our interest concerns the bulk behaviour of the colloidal-rod suspensions, that is, the behaviour far away from the walls of the sample container. The planar boundary conditions, resulting from the repulsive interactions between the rods and the walls, do not affect the phases/states that are observed in the bulk of the suspensions. This is confirmed by experiments that have been preformed with varying sample thickness from 1.3 up to $3 \mathrm{~mm}$. 

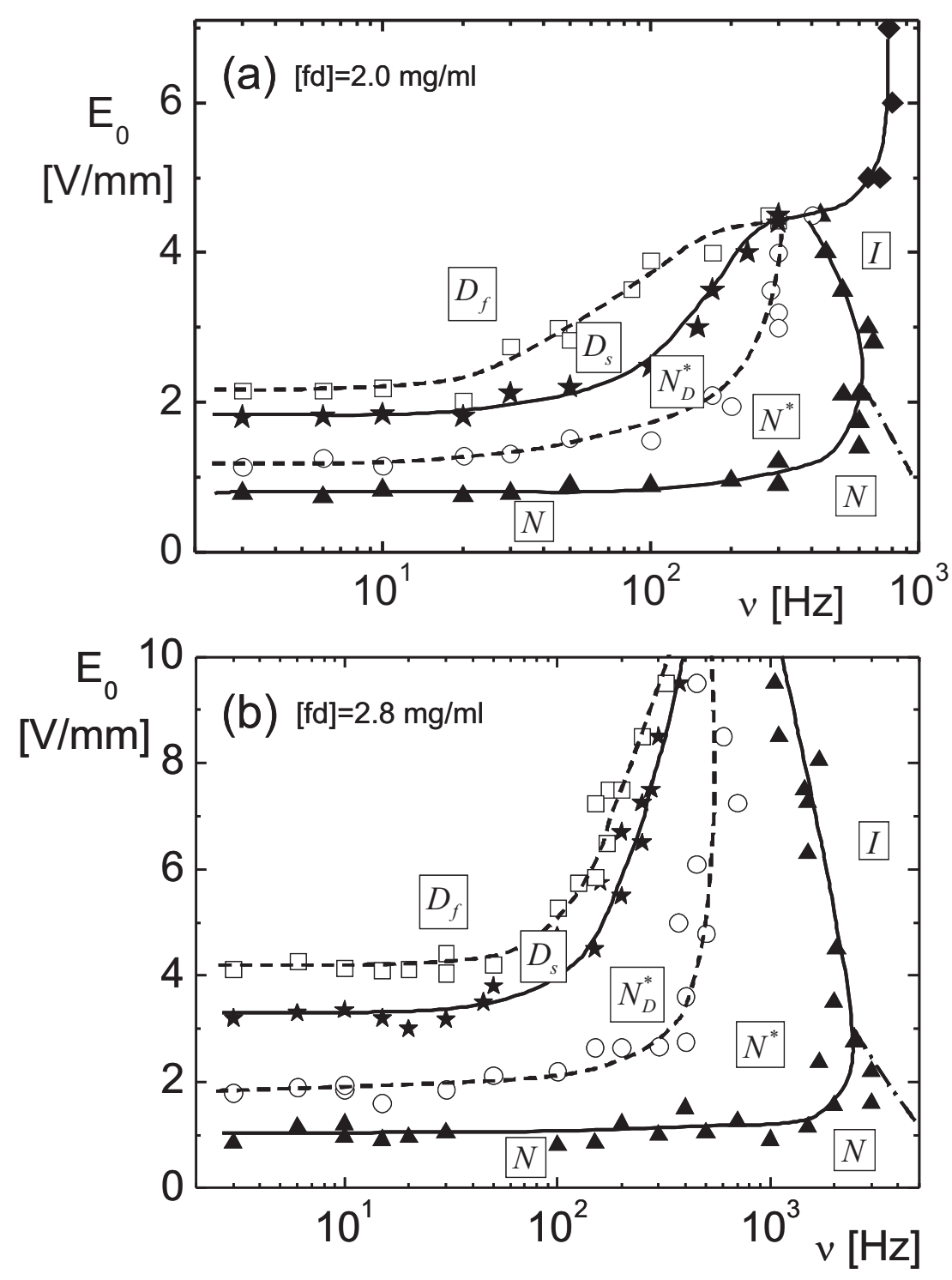

Figure 1: Transition lines in the field-amplitude versus frequency plane for the fd concentration (a) $2.0 \mathrm{mg} / \mathrm{ml}$ and (b) $2.8 \mathrm{mg} / \mathrm{ml}$. The solid lines refer to transitions involving chiral nematic texture while the dashed lines and the dashed-dotted line at high frequencies refer to transitions involving $N$-domains. The various phases/states are discussed in the main text. 


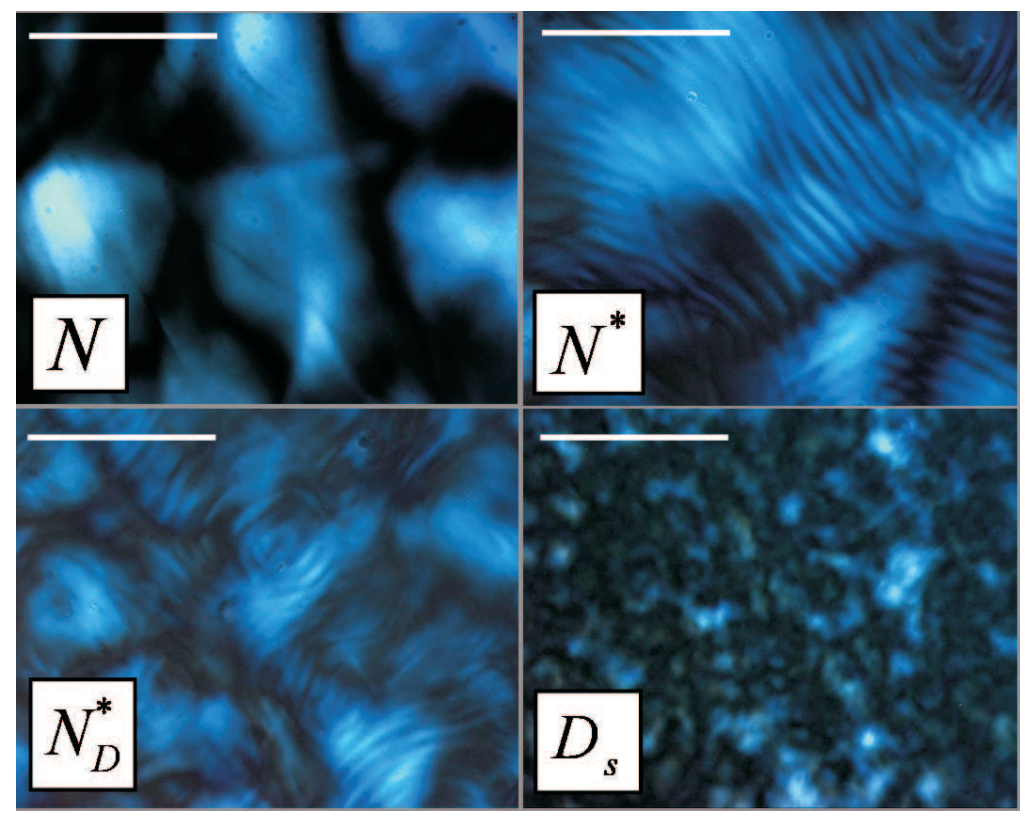

Figure 2: Images of the various phases at low frequency $(10 \mathrm{~Hz})$ on increasing the field amplitude, for $2.8 \mathrm{mg} / \mathrm{ml}$. The scale bar is $200 \mu \mathrm{m}$, and the electric field is perpendicular to the images.

The phase behaviour under electric fields is investigated for fd-virus concentrations of 2.0 and $2.8 \mathrm{mg} / \mathrm{ml}$. The phase diagrams in the field-strength versus frequency plane for these two fd concentrations are given in fig.1. For both concentrations, at low frequencies (say $10 \mathrm{~Hz}$ ) the following transitions are found on increasing the applied electric field amplitude. At zero field and low field amplitudes, the above mentioned coexistence of $N$-domains of non-chiral nematic ordering and isotropic regions is observed. For brevity, we will refer to this state as the $N$-phase and to the non-chiral nematic domains as $N$-domains. A typical image of the $N$ phase is given in fig.2 (left-top). On rotation of the polarizers, a considerable part of the black regions in fig.2 (left top) remain black. This phase is also found without an external field, where there is no preferred direction of domain orientation. Since the probability for a coincidental homeotropic orientation is therefore small, and a considerable part of the black region remains black on rotation of the polarizers, we conclude that this phase is an isotropic-(non-chiral) 
nematic coexistence. The black regions that remain black upon rotation of the polarizers are therefore isotropic, except for the very few nematic domains that happen to have a homeotropic orientation. As can be seen from fig.1, above a threshold field strength, a transition is observed, where, in addition to the existing $N$-domains, a "stripe pattern" is formed, of which a typical morphology is shown in fig.2 (right-top). The stripe pattern can be either a smectic or chiralnematic phase. For a smectic phase, the pitch is of the order of the length of a single rod, which is less than 1 micron for fd virus. However, the observed the pitch is always much larger (at least 10 times larger) as compared to the length of the rods. The stripe pattern can therefore be identified as a chiral-nematic state which will be referred to as the $N^{*}$-state. The same chiral-nematic state is also observed in the absence of an electric field at higher ionic strengths [10],[11],[13]. The chiral-nematic texture extends beyond the $N$-domains and is not nucleated within these domains. Within the $N^{*}$-phase region, there is a coexistence between non-chiral nematic and chiral-nematic texture. On further increasing the field strength, the partly interconnected $N$-domains become smaller and disconnected. We refer to this phase as the $N_{D}^{*}$-phase, where such smaller $N$-domains are present, together with the chiral-nematic texture (the subscript " $D$ " refers to the smaller domains). The typical morphology of the $N_{D}^{*}$-phase is shown in fig.2 (left-bottom). The size of $N$-domains continuously decreases on increasing the field strength. At even higher field amplitudes, the chiral-nematic texture melts, and at the same time the $N$-domains dissolve and are reformed very slowly (on a time scale of tens of seconds). This dynamic state is referred to as the $D_{s}$-state (where "s" stands for slow). A typical instantaneous morphology of the $D_{s}$-state is given in the right-bottom image in fig.2. The time for a $N$-domain to dissolve/reform becomes shorter up to a field strength beyond which it hardly changes anymore. The location in the phase/state diagram beyond which the dissolve/reform time is essentially constant (of the order of a second) is indicated by the upper dashed line in the phase diagram in fig.1. The dynamical state above this transition line is 
referred to here as the $D_{f}$-state (where " $f "$ stands for fast).

In order to quantify the $N$-to- $N^{*}$ - and the $N_{D}^{*}$-to- $D_{s}$-transition, we performed optical pitch measurements and Dynamic Light Scattering (DLS) experiments, respectively.

The variation of the measured $\pi$-pitch in the $N^{*}$ - and $N_{D}^{*}$-phases as a function of the field amplitude for a fixed low frequency of $10 \mathrm{~Hz}$ is shown in fig.3. The pitch is simply measured directly from microscopy images. We did not use Fourier transformation, due to the observed large spread in the measured pitch. Just above the $N$-to- $N^{*}$ transition line (at $\left.1.1 \mathrm{~V} / \mathrm{mm}\right)$, there is a strong variation in the measured pitch, from about $32 \mu \mathrm{m}$ to $12 \mu \mathrm{m}$. The measured $\pi$-pitch is actually the projected pitch of chiral-nematic textures that may not be aligned perpendicular to the electric field. The large spread in the measured pitch at low field strengths may be due to the fact that there is a broad distribution of the orientation of the chiral-nematic director. The largest measured pitch corresponds to a chiral-nematic director that is perpendicular to the electric field, and is the true pitch of the chiral-nematic. The solid line in fig.3 is a guide-to-theeye for the true pitch as a function of the field strength. Note that the pitch seems to diverge at the $N$-to- $N^{*}$ transition, and saturates at higher field amplitudes to about $10 \mu m$, where also the variance of the pitch becomes smaller as compared to low field strengths. The latter indicates that the preferred orientation of the chiral-nematic director is perpendicular to the external field. This complies with the negative birefringence found in Ref.[5]. As can be seen from Fig.3, the true pitch decreases with increasing applied field amplitude. At the $N_{D}^{*}$-to- $D_{s}$ transition, the chiral-nematic texture melts, and at the same time $N$-domains become dynamic in the sense that these domains melt and reform.

Dynamic Light Scattering (DLS) experiments have been performed with a set up where the laser beam is along the electric field, in the vertical direction. The experimental results discussed here are obtained at a scattering angle of $14^{0}$. The corresponding length scale $2 \pi / q$ (with $q$ the scattering wave vector), that is probed by DLS is less than $2 \mu m$. We thus probe 


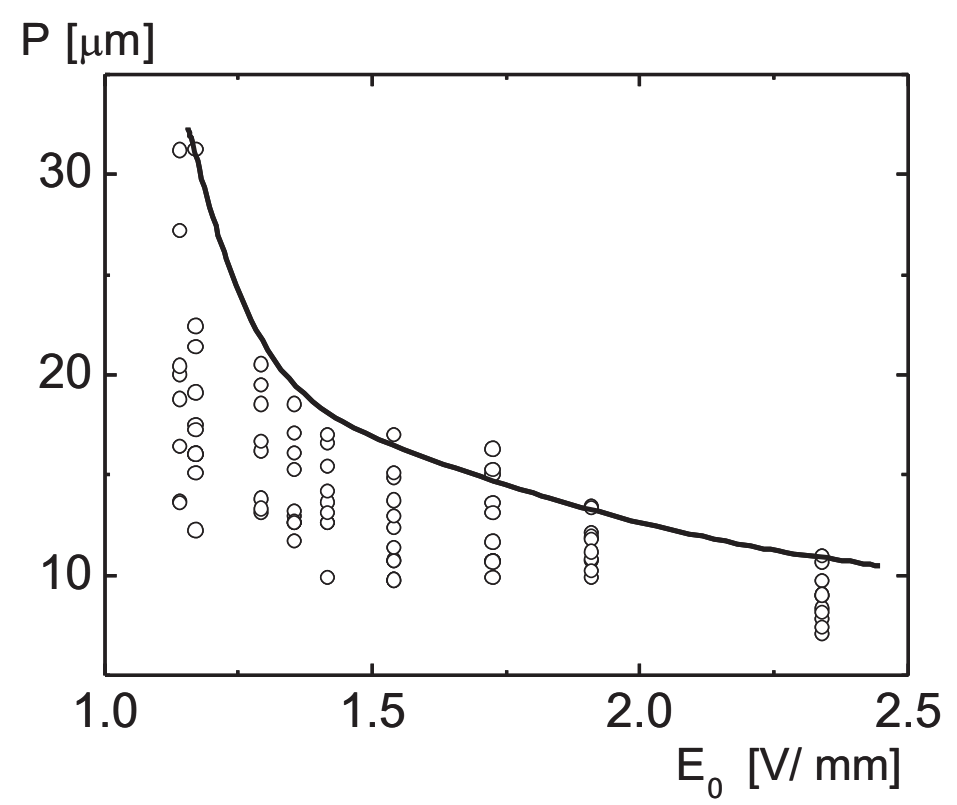

Figure 3: The measured $\pi$-pitch (data points) of chiral-nematic textures for an fd concentration of $2.8 \mathrm{mg} / \mathrm{ml}$ at $10 \mathrm{~Hz}$, as a function of the field amplitude.

the coherent dynamics of single fd-virus particles, in a quite inhomogeneous environment. A detailed interpretation of such DLS data requires an analysis for scattering from a highly inhomogeneous system, including domain interfaces, which is beyond the scope of the present Letter. The goal of these DLS experiments is to show that at the $N_{D}^{*}$-to- $D_{s}$ transition there is a characteristic sudden change in the microscopic dynamics of fd-virus particles. The dynamic light scattering data show a discontinuity of decay times of the light-scattering correlation function at the field strength where the $N_{D}^{*}$-to- $D_{s}$ transition occurs, as shown in fig.4. The observed jump of rod-mobility on crossing the $N_{D}^{*}$-to- $D_{s}$ transition line is in accord with the dynamic behaviour of the $N$-domains as described above. Within the $D_{s}$-state, the decay times are independent of the field strength, within experimental error. However, when the $D_{s}$-to- $D_{f}$ transition line is crossed, decay times decrease with increasing field strength. This corresponds to the observation of faster melting and reforming of small $N$-domains in the $D_{f}$-state.

At high frequencies and high field strengths, the $N^{*}$ texture disappears on increasing the 


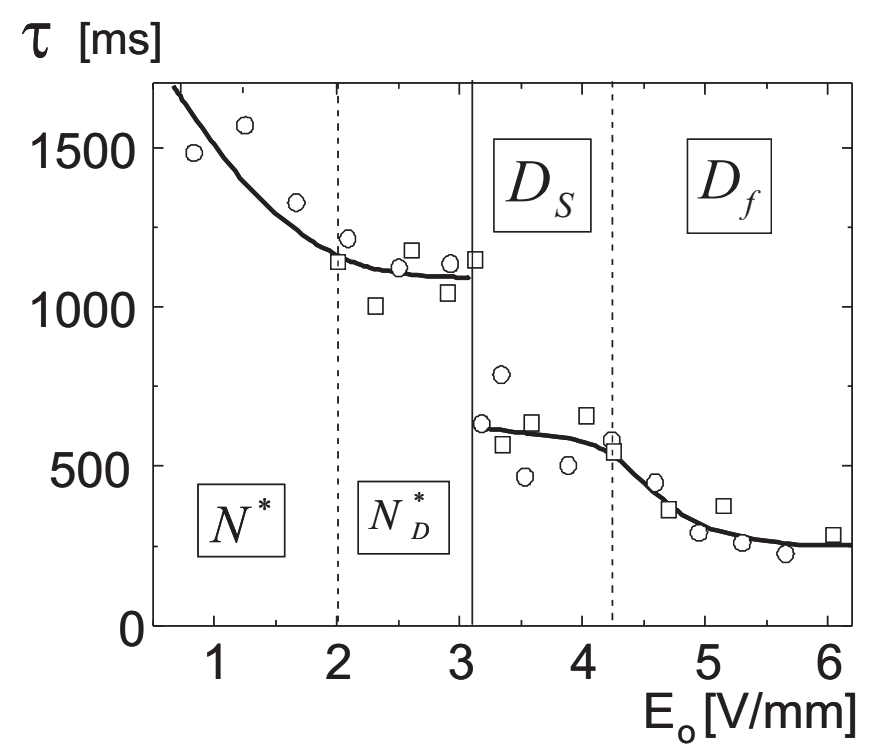

Figure 4: Initial decay times of DLS-correlation functions at a low frequency $(10 \mathrm{~Hz})$ as a function of the field strength, in VV mode at a fixed scattering angle of $14^{0}$ for a fd concentration of $2.8 \mathrm{mg} / \mathrm{ml}$. The direction of the incident laser beam is along the electric field. The experimental error is about $0.2 \mathrm{~s}$. The two different symbols refer to two independent measurements.

frequency, and a homogeneous image is observed (see the left-top image in Fig.5. The same state corresponding to homogeneous images is entered from the $N$-phase on increasing the field strength at high frequency (the dashed-dotted lines in the phase diagrams in Fig.1 are estimates of the location of this transition line). The corresponding state at high frequencies can be either isotropic, or the rods have a homeotropic or planar alignment. In order to detect possible homeotropic or planar alignment, birefringence measurements have been performed with an angle of $15^{0}$ between the laser beam and the vertical direction, along the electric field. No birefringence is observed, which implies that this state is isotropic. We shall therefore refer to this phase as the $I$-phase, standing for the "isotropic phase".

As can be seen from the phase diagram in fig.1a for the fd concentration of $2.0 \mathrm{mg} / \mathrm{ml}$, interestingly, all phase-transition lines merge into a "non-equilibrium critical point" (located at about $300 \mathrm{~Hz}$ and $4.5 \mathrm{~V} / \mathrm{mm}$ ). For the higher fd concentration of $2.8 \mathrm{mg} / \mathrm{ml}$, this "crit- 


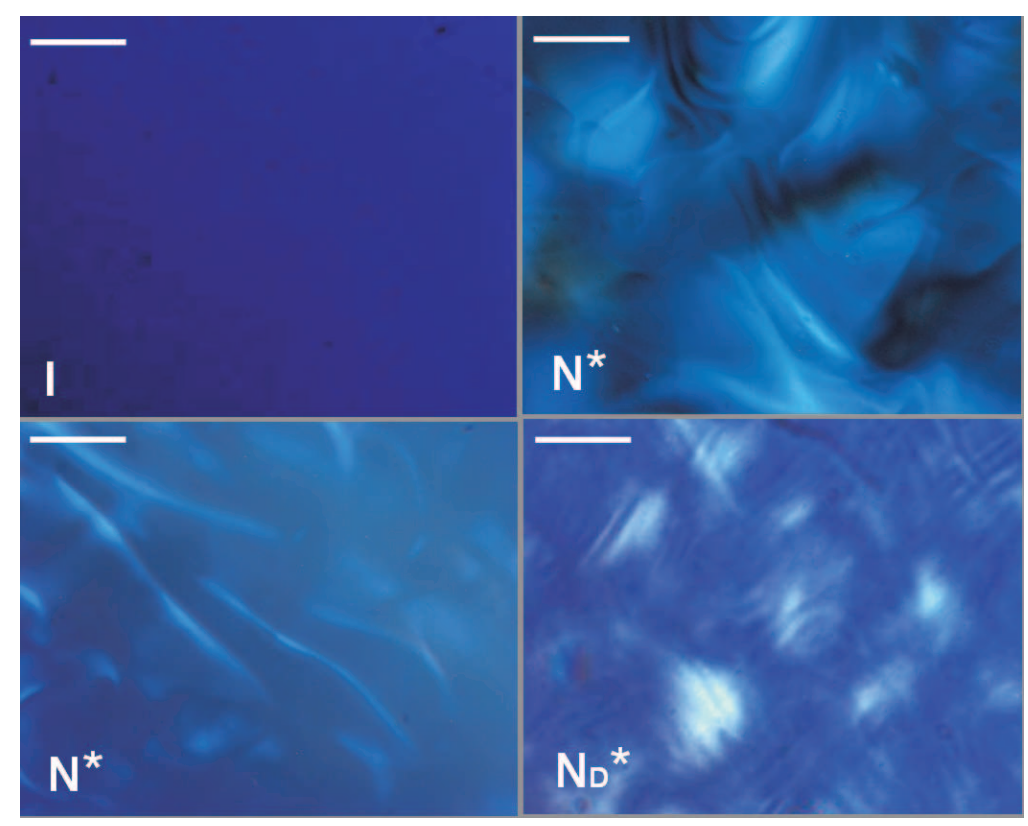

Figure 5: Images of the various phases at a medium field amplitude of $4 \mathrm{~V} / \mathrm{mm}$ on decreasing the frequency (downwards from $4(I), 1.5\left(N^{*}\right), 1.0\left(N^{*}\right)$ to $0.2 k H z\left(N_{D}^{*}\right)$ for the fd concentration $2.8 \mathrm{mg} / \mathrm{ml})$.

ical point" is located at a somewhat higher frequency and at a higher field amplitude (see fig.1b). Measurements beyond field amplitudes of $10 \mathrm{~V} / \mathrm{mm}$ are not included, since these may be affected by heat production. For field amplitudes larger than the "critical amplitude", the $D_{f}$-state transforms directly to the $I$-phase on increasing the frequency (see the $\boldsymbol{\nabla}$ in fig.1a). The right-top and left-bottom images in fig.5 are taken just inside the $N^{*}$-phase, where chiralnematic texture is beginning to develop, and deeper inside the $N^{*}$-phase, respectively. Comparing fig.5 (left-bottom) with the right-top image in fig.2 for low frequencies reveals a very much larger pitch and more irregular chiral-nematic texture for these higher frequencies. On further decreasing the frequency, the $N$-domains become smaller and more regular at the $N^{*}$ to- $N_{D}^{*}$ transition line, similar to the lower frequencies on increasing the field amplitude. The right-bottom image in fig. 5 corresponds to the $N_{D}^{*}$-phase.

On applying an electric field to an isotropic solution (with a concentration below $1.5 \mathrm{mg} / \mathrm{ml}$ ), 
no nematic state could be established. This clearly shows that double-layer polarization does not induce an isotropic-to-nematic or any other kind of transition.

It should be mentioned that there is no macroscopic flow, not in the $N^{*}$ and $N_{D}^{*}$ states, nor in the dynamical $D_{s}$ and $D_{f}$ states. The patterns in the $N^{*}$ and $N_{D}^{*}$ states are static, while in the dynamical $D_{s}$ and $D_{f}$ states the nematic domains melt and reform at a fixed position. Accidental dust particles either do not move at all (in the $N^{*}$ and $N_{D}^{*}$ states) or move only over limited distances smaller than the size of $\mathrm{N}$ domains (in the $D_{s}$ and $D_{f}$ states), which are due to structural rearrangements and/or local electro-osmotic flows.

In order to quantitatively describe the observed phases and dynamical states, a theory should be developed that includes an analysis of (i) the double-layer polarization due to the external field, (ii) interactions between rods arising from double-layer polarization and the accompanied electro-osmotic flows, and (iii) the phase behaviour that is a consequence of such interactions. As yet such a theory for double-layer polarization induced phase/state transitions does not exist. Part of the above described transitions can be understood qualitatively as follows. Consider first a weak, zero-frequency electric field. In this case, ion currents are small so that ions reside within the double layer for relatively long times. During the relatively long time needed for an ion to pass an fd-virus particle, it responds to the electric field imposed by the surface charges of the fd-virus particle. This leads to slight polarization of the double layer. For such low field strengths, the field-induced dipole moment will increase with increasing field strength. The dipolar interactions enhance the tendency of rods to align and re-orient, where electro-osmotic flows might also play a role. At sufficiently high degree of orientational order, the chiral-nematic structure of the cores of the individual rods leads to a stable chiralnematic state. At higher field strengths, however, ion velocities are large, so that ions reside in the vicinity of an fd-virus particle for a relatively short time. The ions can therefore not respond to the field imposed by the surface charges of the rods so that the double-layer is 
destroyed. This explains melting of the chiral-nematic phase on increasing the field strength at higher field amplitudes and low frequencies. On subsequently increasing the frequency, this molten state will transit to a chiral nematic again. The reason for this is that ions will reside within the double layer on increasing the frequency. The ions are now typically displaced over distances, during a cycle of the external electric field, comparable to the linear dimension of double-layer thickness in the direction of the field. The double-layer structure is now restored and polarized on increasing the frequency of the external field. Again, the resulting dipolar interactions stabilize the chiral nematic. At very high frequencies the double-layer structure is expected to be undistorted, and one would expect the same behaviour as in the absence of the electric field. It is therefore not clear why the $N$-domains (which are also formed in the absence of an electric field) are not stable anymore at very high frequencies, and instead an isotropic state is observed. This might be due to electro-osmotic flows which are present in the $I$-state but not in the $N$-phase at zero field strength. These arguments remain speculative as

long as a quantitative theory for double-layer polarization and the resulting interactions and phase transitions is not available.

Acknowledgement This work has been supported by the Deutsche Forschungsgemeinschaft (Trans-regio SFB 6018 "Physics of Colloidal Dispersions in External Fields").

\section{References}

[1] P.G. de Gennes, J. Prost, The Physics of Liquid Crystals (Carendon Press, London) 1993.

[2] U. Dassanayake, S. Fraden, A. van Blaaderen, J. Chem. Phys. 112 (2002) 3851.

[3] A. Yethiraj, A. van Blaaderen, Nature (London) 421 (2003) 513.

[4] F. Mantegazza, T. Bellini, M. Buscaglia, V. Degiorgio, D.A. Saville, J. Chem. Phys. 113 (2000) 6984. 
[5] Kramer, H., Graf, C., Hagenbuchle, M., Johner, C., Martin, C., Schwind, P., Weber, R., J. Phys. II France, 4 (1994) 1061.

[6] F. Mantegazza, M. Caggioni, M.L. Jiménez, T. Bellini, Nature Physics 1 (2005 103.

[7] D. Saintillan, E. Darve, E.S.G. Shaqfeh, J. Fluid Mech. 563 (2006) 223.

[8] X. Schlagberger, R.R. Netz, Eur. Phys. Lett., submitted.

[9] H. Isambert, A. Ajdari, J.-L. Viovy, J. Prost, Phys. Rev. E 56 (1997) 5688.

[10] S. Fraden in Observation, Prediction and Simulation of Phase Transitions in Complex Fluids, edited by M. Baus, L.F. Rull, J.P. Ryckaert (Kluwer Academic Publishers, Dordrecht) NATO-ASI-Series C, vol. 460, 1995, page 113.

[11] Z. Dogic, S. Fraden, Langmuir 16 (2000) 7820.

[12] E. Grelet, S. Fraden, Phys. Rev. Lett. 90 (2003) 198302.

[13] Z. Dogic, S. Fraden, Phil. Trans. R. Soc. Lond. A, 359 (2001) 997. 